\title{
CLASS NUMBERS OF TOTALLY POSITIVE BINARY FORMS OVER TOTALLY REAL NUMBER FIELDS ${ }^{1}$
}

\author{
BY JIH-MIN SHYR \\ Communicated by Olga Taussky Todd, October 30, 1976
}

Let $(V, q)$ be a totally positive binary quadratic space over a totally real number field $k$. A lattice in $V$ is a finitely generated $D_{k}$-submodule of $V$ of rank $2, \omega_{k}$ being the ring of integers in $k$. We can define the notions of class and genus on the set of all lattices in $V$ (cf. [1]). The purpose of this note is to announce an explicit formula for the number of proper classes in the genus of any free lattice in $V$. The details will be published elsewhere.

1. A class number relation. Scaling $q$ by a constant factor if necessary, we may assume that $q$ represents 1 . Then, the binary quadratic space $(V, q)$ is $k$ isomorphic to $(k(\sqrt{-} \delta), N), \delta$ being the discriminant of $(V, q)$ and $N$ the norm of $k(\sqrt{-} \delta)$ to $k$. Let $G$ be the kernel of the norm map $\nu: R_{K / k}\left(\mathbf{G}_{m}\right) \rightarrow \mathbf{G}_{m}$, where $K=k(\sqrt{-} \delta), R_{K / k}$ is the Weil functor of restricting the field of definition from $K$ to $k$ (cf. [6]) and $\mathbf{G}_{m}$ denotes the multiplicative group of nonzero elements in a universal domain containing $k$. Then, the algebraic torus $G$ is nothing but the special orthogonal group of $N$, and the class number $H$ of $G$ over $k$, which is intrinsically defined, can be interpreted as the number of proper classes in the genus of any free lattice in $K$.

Consider the isogeny $\lambda: R_{K / k}\left(\mathbf{G}_{m}\right) \rightarrow G \times \mathbf{G}_{m}$ defined by

$$
\lambda(x)=\left(x^{2} v(x)^{-1}, \nu(x)\right) \text {. }
$$

If we identify the character groups of $R_{K / k}\left(\mathbf{G}_{m}\right), G, \mathbf{G}_{m}$ by $\mathbf{Z}[\&], \mathbf{Z}[\&] / Z s, \mathbf{Z}$, respectively, \& being the Galois group of $K / k$ and $s=\Sigma_{\sigma \in \infty} \sigma$, then the dual $\hat{\lambda}$ : $\widehat{G \times G_{m}} \rightarrow \widehat{R_{K / k}\left(G_{m}\right)}$ of $\lambda$ is given by

$$
\hat{\lambda}(\gamma \bmod \mathbf{Z} s, z)=z s+(2 \gamma-S(\gamma) s),
$$

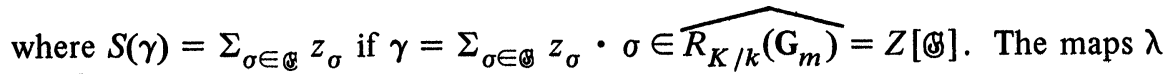
and $\hat{\lambda}$ induce naturally the following maps: $\lambda_{v}: R_{K / k}\left(\mathbf{G}_{m}\right)_{v} \rightarrow\left(G \times \mathbf{G}_{m}\right)_{v}$ for each (finite or infinite) prime $v$ of $k, \lambda_{k}^{c}: R_{K / k}\left(\mathbf{G}_{m}\right)_{k}^{c} \rightarrow\left(G \times \mathbf{G}_{m}\right)_{p}^{c}$ for each finite prime $p$ of $k, \lambda_{k}^{\infty}: R_{K / k}\left(\mathbf{G}_{m}\right)_{k}^{\infty} \rightarrow\left(G \times \mathbf{G}_{m}\right)_{k}^{\infty}$, and $(\hat{\lambda})_{k}: \widehat{\left(G \times \mathbf{G}_{m}\right)_{k}} \rightarrow$ $\widehat{R_{K / k}\left(\mathbf{G}_{m}\right)_{k}}$ (cf. [2]), $\infty$ being the set of all infinite primes of $k$. For a

AMS (MOS) subject classifications (1970). Primary 12A50, $20 \mathrm{G} 30$.

${ }^{1}$ For the unexplained notions, see $[2],[3],[5]$. 
homomorphism $\alpha$ of commutative groups with finite kernel and cokernel, we define the $q$-symbol of $\alpha$ by $q(\alpha)=[\operatorname{Cok} \alpha] /[\operatorname{Ker} \alpha]$. Then the $q$-symbols of above maps are defined; moreover, we have $q\left(\lambda_{p}^{c}\right)=1$ for almost all $\downarrow$. In [5], we obtain the following class number relation:

$$
H=\frac{h_{K}}{h_{k}} \cdot \frac{\left.\tau \cdot q(\hat{\lambda})_{k}\right) q\left(\lambda_{k}^{\infty}\right)}{\Pi_{v \in \infty} q\left(\lambda_{v}\right) \cdot \Pi_{k} q\left(\lambda_{k}^{c}\right)},
$$

where $\tau$ is the Tamagawa number of $G$ over $k$, and $h_{K}$ (resp. $h_{k}$ ) is the class number of $K$ (resp. $k$ ). By a result of Ono on the Tamagawa numbers of algebraic tori, we have $\tau=[K: k]=2$ (cf. [3]).

2. Computation of $q$-symbols. The computations for $\left.q(\hat{\lambda})_{k}\right)$ and $\Pi_{v \in \infty} q\left(\lambda_{v}\right)$ are elementary; we have $\left.q(\hat{\lambda})_{k}\right)=1, \Pi_{v \in \infty} q\left(\lambda_{v}\right)=1$. From Dirichlet's unit theorem we obtain $q\left(\lambda_{k}^{\infty}\right)=2^{n} /\left[U_{K}^{0}: U_{k}^{0}\right]$, where $n=[k: Q]$ and $U_{K}^{0}$ (resp. $U_{k}^{0}$ ) is the torsion free subgroup of the unit group $U_{K}$ (resp. $U_{k}$ ) of $K$ (resp. $k$ ). Since the degree of $\lambda$ is 2 , we have $q\left(\lambda_{k}^{c}\right)=1$ if $\not \nmid 2$ and $\npreceq \Varangle d_{K / k}$ $=$ the relative discriminant of $K / k$ (cf. [2]). The remaining $q\left(\lambda_{k}^{c}\right)$ can be computed by means of the local norm index theorem in class field theory. We have the following lemma:

LEMMA.

$$
\begin{aligned}
& q\left(\lambda_{k}^{c}\right)=2 \quad \text { if } k \mid d_{K / k} \text { and } p \times 2 \text {, } \\
& =2^{e(p / 2) f(p / 2)+1} \text { if } p \mid d_{K / k} \text { and } p \mid 2 \text {, } \\
& =2^{e(p / 2) f(p / 2)} \quad \text { if } k\left\langle d_{K / k} \text { and } p / 2\right. \text {, }
\end{aligned}
$$

where $e(p / 2)$ and $f(k / 2)$ denote the ramification index and the residue class degree of $k_{p} / \mathbf{Q}_{2}$, respectively.

Therefore, (1) can be simplified as

$$
H=\frac{h_{K}}{h_{k}} \cdot \frac{2^{1-r}}{\left[U_{K}^{0}: U_{k}^{0}\right]},
$$

$r$ being the number of ramified primes in $K / k$.

3. An explicit formula for $H$. First, we shall describe a recent result of Shintani concerning the relative class number $h_{K} / h_{k}$ (cf. [4]). Let $\chi$ be the quadratic character of the narrow ideal class group of $k$ with the conductor $d_{K / k}$ and associated to $K / k$ in class field theory. Take a complete set of representatives $\mathfrak{I}_{1}, \ldots, \mathfrak{l}_{h}$ of the ideal classes of $k$ such that each $\mathfrak{U}_{m}$ is integral and prime to $d_{K / k}$. For $x \in k$, we denote by $x^{(1)}, \ldots, x^{(n)}$ the $n$ conjugates of $x$ over $\mathbf{Q}$. If we imbed $k$ into $\mathbf{R}^{n}$ by $x \rightarrow\left(x^{(1)}, \ldots, x^{(n)}\right)$, then $k^{\times}$acts on $\mathbf{R}^{n}$ as a group of linear transformations via the componentwise multiplication. Moreover, $\left(\mathbf{R}_{+}^{\times}\right)^{n}$ is invariant under the above action by the group $U_{k}^{+}$of totally positive units in $k$; if we denote by $C\left(v_{1}, \ldots, v_{i}\right)$ the $i$-dimensional open simplicial cone $\left\{t_{1} v_{1}+\cdots+t_{i} v_{i} ; t_{1}, \ldots, t_{i} \in \mathbf{R}_{+}^{\times}\right\}$generated by $\mathbf{R}$-linearly independent 
vectors $v_{1}, \ldots, v_{i}$ in $\mathbf{R}^{n}$, we have

$$
\left(\mathbf{R}_{+}^{\times}\right)^{n}=\bigcup_{j \in J} \bigcup_{u \in U_{k}^{+}} u C_{j}\left(v_{j 1}, \ldots, v_{j i(j)}\right) \quad \text { (disjoint union), }
$$

where $J$ is a finite set and $v_{j 1}, \ldots, v_{j i(j)} \in \bigcap_{k} \cap\left(\mathbf{R}_{+}^{\times}\right)^{n}$. The relative class number $h_{K} / h_{k}$ is given by

$$
2^{n} w_{K}\left[U_{k}: U_{k}^{+}\right]^{-2}\left[U_{k}^{+}: N\left(U_{k}\right)\right]^{-1} \sum_{m=1}^{h} A_{m}(\chi)
$$

with $w_{K}=$ the number of roots 1 contained in $K$ and

$$
\begin{aligned}
A_{m}(\chi)= & \chi\left(\mathfrak{U}_{m}\right)^{-1} \sum_{j \in J} \sum_{x} \sum_{z_{1}, \ldots, z_{i(j)}=0}^{f-1} \chi\left(\sum_{p=1}^{i(j)}\left(z_{p}+x_{p}\right) v_{j p}\right) \\
& \times \frac{(-1)^{i(j)}}{n} \sum_{l} \prod_{p=1}^{i(j)} \frac{B_{l_{p}}\left(\left(z_{p}+x_{p}\right) / f\right)}{l_{p} !} \operatorname{tr}\left(\prod_{p=1}^{i(j)} v_{j p}^{l_{p}-1}\right),
\end{aligned}
$$

where $x$ ranges on the finite set $R\left(j, \mathfrak{U}_{m}\right)$ of all $i(j)$-tuples $x=\left(x_{1}, \ldots, x_{i(j)}\right)$ of rational numbers satisfying $0<x_{p} \leqslant 1(p=1,2, \ldots, i(j))$ and $\Sigma_{p=1}^{i(j)} x_{p} v_{j p}$ $\in \mathfrak{Z}_{m}, f$ is the smallest positive integer such that $f v_{j p} \in d_{K / k}$ for all $j \in J$ and $1 \leqslant p \leqslant i(j), l$ is taken over all $i(j)$-tuples $l=\left(l_{1}, \ldots, l_{i(j)}\right)$ of nonnegative integers such that $l_{1}+l_{2}+\cdots+l_{i(j)}=i(j), B_{j}$ denotes the usual $j$ th Bernoulli polynomial, and $\operatorname{tr}(x)=x_{1}+\cdots+x_{n}$ if $x=\left(x_{1}, \ldots, x_{n}\right) \in \mathbf{R}^{n}$. Upon employing this result, we have the following explicit formula for $H$ :

THEOREM. The class number $H$ is given by

$$
H=\frac{w_{K} 2^{1-r}}{\left[U_{k}: U_{k}^{+}\right]} \sum_{m=1}^{h_{k}} A_{m}(\chi) .
$$

\section{REFERENCES}

1. O. T. O'Meara, Introduction to quadratic forms, 2nd. printing, corrected, SpringerVerlag, Berlin and New York, 1971. MR 50 \#269.

2. T. Ono, Arithmetic of algebraic tori, Ann. of Math. (2) 74 (1961), $101-139$. MR 23 \#A1640.

3. - On the Tamagawa number of algebraic tori, Ann. of Math. (2) 78 (1963), 47-73. MR 28 \#94.

4. T. Shintani, On evaluation of zeta functions of totally real algebraic number fields at nonpositive integral places (to appear).

5. J. Shyr, On some class number relations of algebraic tori, Michigan Math. J. (to appear).

6. A. Weil, Adeles and algebraic groups, Lecture notes, Inst. for Advanced Study, Princeton, N. J., 1961.

SCHOOL OF MATHEMATICS, INSTITUTE FOR ADVANCED STUDY, PRINCETON, NEW JERSEY 08540

DEPARTMENT OF MATHEMATICS, UNIVERSITY OF GEORGIA, ATHENS, GEORGIA 30602 